\title{
Numerical continuation methods for
}

\section{nonlinear equations and bifurcation problems}

\section{James P. Abbott}

This thesis investigates some aspects of the continuation method for the solution of a system of nonlinear equations, $f(x)=0$, $f: D \subset R^{n} \rightarrow R^{n}$. This approach is useful for generating methods which do not rely on a good initial estimate of a solution and the problem is converted to one of following the solution trajectory $x(t)$ of a problem of the form $H(x(t), t)=0, H: D \subset R^{n} \times R \rightarrow R^{n}$, from the starting guess $x_{0}=x(0)$, hopefully to the solution $x^{*}$.

In Chapter 1 we give a brief introduction and note that $x(t)$ also satisfies

$$
\dot{x}(t)=-\partial_{x} H(x, t)^{-1} \partial_{t} H(x, t), x(0)=x_{0},
$$

and so we can follow $x(t)$ by applying methods traditionally used for the solution of ordinary differential equations. In Chapter 2 we consider general single-step methods and, in particular, Runge-Kutta methods, for following $x(t)$. We also give conditions on the methods to attain rapid convergence to $x^{*}$ and, as a result, for a particular choice of $H(x, t)$ we are able to derive methods which have improved rates of convergence to $x^{*}$. We apply similar arguments in Chapter 3 to the class of linear multistep methods and again generate methods which follow $x(t)$ accurately and then give rapid final convergence to $x^{*}$.

In Chapter 4 we consider Newton-like methods for finding $x\left(t_{i}\right)$ for a

Received 27 July 1977. Thesis submitted to the Australian National University, April 1977. Degree approved, August 1977. Supervisor: Dr Richard P. Brent. 
sequence of values $\left\{t_{i}\right\}$, and discuss the accuracy and computational efficiency of the methods. We use the results of Chapter 2 to derive a method which changes in a continuous way from one which follows $x(t)$ accurately to one which converges rapidly to $x^{*}$.

Chapter 5 is concerned with problems where the need to follow the solution of $H(x(t), t)=0$ arises naturally. We consider, in particular, the difficulties associated with certain critical points, that is, points on the solution branch $(x(t), t)$ at which $\partial_{x} H(x, t)$ is singular. We describe an efficient method for following a branch through a simple. turning point and present an efficient method for determining such turning points accurately. This method is also useful for finding certain simple bifurcation points.

Finally, in Chapter 6 , we consider the problem of finding several solutions of the equation $f(x)=0$. We consider two recent approaches and show that the two methods are essentially the same. A reformulation of one of the methods indicates a technique which is, in some sense, more efficient than the other methods. 